\title{
LIE ISOMORPHISMS OF PRIME RINGS
}

\author{
BY \\ WALLACE S. MARTINDALE III
}

1. Introduction. A Lie isomorphism $\phi$ of a ring $S$ onto a ring $R$ is a one-one additive mapping of $S$ onto $R$ which preserves commutators, i.e.,

$$
\begin{aligned}
\phi(x+y) & =\phi(x)+\phi(y), \\
\phi(x y-y x) & =\phi(x) \phi(y)-\phi(y) \phi(x)
\end{aligned}
$$

for all $x, y \in S$. Our interest and viewpoint toward the study of Lie isomorphisms of rings was originally (and still is) inspired by the work done by I. N. Herstein on generalizing classical theorems on the Lie structure of total matrix rings to results on the Lie structure of arbitrary simple rings. In our case the starting point was the realization that it should be possible to extend the following theorem of L. Hua [1]: every Lie automorphism of the ring $R$ of all $n \times n$ matrices over a division ring, $n>2$, characteristic $\neq 2,3$, is of the form $\sigma+\tau$, where $\sigma$ is either an automorphism or the negative of an antiautomorphism of $R$ and $\tau$ is an additive mapping of $R$ into its center which maps commutators into zero. Indeed, using some of Hua's techniques and some valuable suggestions due to Nathan Jacobson, we were in [2], roughly speaking, able to obtain the same conclusion under the weaker assumption that $R$ was merely a primitive ring possessing three orthogonal idempotents whose sum was 1 . In a recent paper [3], while making the stronger assumption that $R$ was simple, we were able to lower the number of idempotents from three to two. For the most part, the same techniques were used in this second paper, although a tensor product method due to Jacobson was to replace tedious calculations involving matrix units due to Hua, and some results of Herstein on Lie ideals of simple rings seemed necessary.

Our goal in this paper is Theorem 11, in which we extend the above results to the situation where $R$ is a prime ring with two orthogonal idempotents whose sum is 1 . Primeness is a natural generalization of simplicity and primitivity, and, in the sense of keeping free of the radical and of (sub)direct sums of ideals, it is perhaps the strongest generalization one may make. Whether the assumption of idempotents is necessary or not is still a major open question. In all our work on the subject (including the present paper) our arguments rest heavily on the presence of a nontrivial idempotent. A successful removal of the assumption of idempotents would certainly require totally new methods; one would, for example, have to face the situation of an arbitrary division ring.

Received by the editors July 31, 1968. 
A more accurate statement of our result for Lie isomorphisms of primitive rings would reveal the fact that the image of $R$ under the (anti)isomorphism $\sigma$ (and hence under $\tau$ ) may in general be contained in a larger ring than $R$ itself. Indeed, an example given in [2, p. 916], shows that the image of $\sigma$ need not be contained in $R$. Fortunately, in this case we could consider $R$ as a "dense" subring of the ring of all linear transformations of a vector space over a division ring. So, although we still could (and did) use most of the techniques as in [3] for the case where $R$ is a prime ring, we were confronted with the new problem of finding a suitable extension ring of $R$ which would contain the images of $\sigma$ and $\tau$.

A choice for an extension ring of $R$ which proves successful for the prime ring case is the so-called complete ring of right quotients $Q$ of $R$. A good account of the construction and properties of this ring is given by Lambek in [4, Chapter 4]. In $\$ 2$ of this paper we give our version (basically Utumi's formulation of $Q$ ) of how we feel $Q$ should be characterized. The remainder of the section is devoted to the problem of, given a prime ring $R$ with complete ring of right quotients $Q$, and given several rings closely related to $R$, the determination of the complete ring of right quotients of these related rings in terms of $Q$. The results we obtain, Theorems 1 , 2 , and 3, are very straightforward, and, although we have not explicitly seen them stated in the literature, we believe that they are all more or less well known. For completeness, however, we provide the details of the proofs.

In $\$ 3$ a key result (Theorem 4) and useful corollary (Theorem 5) are proved. Theorem 4 says that in a prime ring $R$, if $a x b=b x a$ for all $x \in R$, then $a$ and $b$ are $C$-dependent, where $C$ is the center of the complete ring of right quotients $Q$. This generalizes to prime rings a result of Amitsur [5, p. 215, Lemma 6(a)] for primitive rings. Theorem 5 , a theorem on tensor products, has as an important application (pointed out first to the author by Jacobson), Theorem 6, which describes idempotents in terms of the Lie product.

The study of Lie isomorphisms proper is begun in $\$ 4$. Theorem 7, which describes what happens to idempotents under Lie isomorphisms, splits our problem into what we call Case 1 and Case 2. Theorem 8, in which Herstein's Lie theory of simple rings appears, reduces each case into two further cases (Cases 1a, 1b, 2a, 2b). The remainder of this section is devoted to the proof of Theorem 9, the main theorem for Case 1a.

In $\S 5$, the main theorem for Case $1 b$, Theorem 10, is first worked out. A device (first mentioned to us by Jacobson) reduces Case 2 to Case 1, and so we quickly reach Theorem 11, the main theorem of our paper. Theorems 12, 13, and 14 then show that previous results of ours on Lie isomorphisms of simple and primitive rings follow as corollaries of Theorem 11 .

2. The complete ring of quotients of a prime ring. The notion of a complete ring of right quotients of an arbitrary ring $R$ has been given by Lambek and Findley [6], Utumi [7], and others. We choose to use the formulation due to 
Utumi and proceed to review some of the relevant definitions. A right ideal $D$ of a ring $R$ is said to be dense if, for any given pair of ring elements $r_{1}, r_{2}$ with $r_{1} \neq 0$, there exists an $r \in R$ such that $r_{1} r \neq 0$ and $r_{2} r \in D$. If $D$ and $D^{\prime}$ are dense right ideals of $R, J$ is a right ideal of $R$ containing $D$, and $a \in R$, then the sets $J, D \cap D^{\prime}$, and $a^{-1}=\{x \in R \mid a x \in D\}$ are easily seen to be dense right ideals. Let $W(R)$ $=\{x \in R \mid x D=0$ for some dense right ideal $D\}$. One shows, using the preceding remark, that $W(R)$ is a two-sided ideal of $R$. The relevance of $W(R)$ will be evident after we now give the characterizing properties of the complete ring of right quotients $Q(=Q(R))$ of a ring $R$.

Definition. A ring $Q$ is said to be a complete ring of right quotients of a ring $R$ if:

(1) $R \subseteq Q$.

(2) If $f \in \operatorname{Hom}_{R}(D, R)$, where $D$ is a dense right ideal of $R$, then there exists $q \in Q$ such that $q d=f(d)$ for all $d \in D$.

(3) If $q \in Q$, there is a dense right ideal $D$ of $R$ such that $q D \subseteq R$.

(4) For all $q \in Q, q=0$ if and only if $q D=0$ for some dense right ideal $D$ of $R$.

We now state (and outline part of the proof of) the following remark: $Q$ exists if and only if $W(R)=0$ and is unique up to isomorphism.

For a construction of $Q$ one proceeds by first forming the set

$$
P=\bigcup\left\{\operatorname{Hom}_{R}(D, R) \mid D \text { dense right ideal of } R\right\}
$$

and defining elements $f$ (acting on $D$ ) and $g$ (acting on $D^{\prime}$ ) of $P$ to be equivalent if $f=g$ on $D \cap D^{\prime}$. Let $Q$ then be the set of equivalence classes. Addition and multiplication are basically given by the usual addition and composition of representatives acting on suitably restricted domains. We show first that $R$ may be isomorphically embedded in $Q$ (and then, for simplicity, identify $R$ with its isomorphic image). Indeed, consider the mapping $a \rightarrow \hat{a}_{l}$ of $R$ into $Q$, where $\hat{a}_{l}$ is the equivalence class determined by the left multiplication $a_{l}$ acting on $R$. Then $\hat{a}_{l}=0$ means that $a D=0$ for some dense right ideal $D$ of $R$, and so $a=0$ since $W(R)=0$. The proofs of properties (2), (3), and (4) are straightforward, with $W(R)=0$ again being needed to show (4). Conversely, property (4) certainly implies that $W(R)=0$.

Next suppose that $x_{1} \rightarrow x_{2}$ is an isomorphism of $R_{1}$ onto $R_{2}$, and suppose that $Q_{1}$ and $Q_{2}$ are complete rings of right quotients of $R_{1}$ and $R_{2}$, respectively. If $\hat{f}_{1} \in Q_{1}$, with $f_{1}: x_{1} \rightarrow y_{1}$ an $R_{1}$-homomorphism of $D_{1}$ into $R_{1}$, then $f_{2}: x_{2} \rightarrow y_{2}$ is easily seen to be an $R_{2}$-homomorphism of the dense right ideal $D_{2}$ into $R_{2}$. Using the fact that $W(R)=0$, one shows that $\hat{f}_{1} \rightarrow \hat{f}_{2}$ gives the desired ring isomorphism of $Q_{1}$ onto $Q_{2}$.

The condition $W(R)=0$ is satisfied in particular whenever the so-called right singular ideal $Z(R)=0$, since every dense right ideal is essential. We are also assured that $W(R)=0$ if $1 \in R$. In fact, suppose $a D=0$ for some dense right ideal 
$D$ of $R$, where $a \neq 0$. Then there exists $r \in R$ such that $a r \neq 0$ but $1 \cdot r=r \in D$, a contradiction.

We now develop several results which indicate the connection between the complete ring of quotients of a ring $R$ and the complete ring of quotients of a ring related to $R$. We do not assume that $1 \in R$. Although these results are undoubtedly known, we have not seen explicit statements of them and therefore will provide proofs.

Lemma 1. Let $0 \neq U$ be an ideal of a prime ring $R$, and let $f \in \operatorname{Hom}_{U}(D, U)$, where $D$ is a dense right ideal of $U$. Then $D U$ is a dense right ideal of $R$ and $f \in \operatorname{Hom}_{R}(D U, R)$.

Proof. Let $r_{1} \neq 0, r_{2} \in R$. Since $R$ is prime $r_{1} u \neq 0$ for some $u \in U$. Because $D$ is dense in $U$, there exists $v \in U$ such that $\left(r_{1} u\right) v \neq 0$ and $\left(r_{2} u\right) v \in D$. Again because $R$ is prime there exists $w \in U$ such that $r_{1}(u v w) \neq 0$. Since $r_{2} u v w \in D U$, we have shown that $D U$ is a dense right ideal of $R$. For $d \in D, u \in U, r \in R$ note that

$$
f((d u) r)=f(d(u r))=f(d)(u r)=f(d u) r,
$$

i.e., $f \in \operatorname{Hom}_{R}(D U, R)$.

COROLlaRY. $U$ is a dense right ideal of $R$.

THEOREM 1. Let $0 \neq U$ be an ideal of a prime ring $R$. Then $Q(U)=Q(R)$.

Proof. Let $f \in \operatorname{Hom}_{U}(D, U)$, where $D$ is a dense right ideal of $U$. By Lemma 1, $D U$ is a dense right ideal of $R$ and $f \in \operatorname{Hom}_{R}(D U, R)$. Then there exists $q \in Q(R)$ such that $q d u=f(d u)$ for all $d \in D$ and $u \in U$. But $f(d u)=f(d) u$, and so $(q d-f(d)) U$ $=0$, which forces $q d=f(d)$, by property (4). This completes the proof of (2). Next, if $q \in Q(R)$, there is a dense right ideal $D$ of $R$ such that $q D \subseteq R$. If $u_{1} \neq 0$, $u_{2} \in U$, there exists $r \in R$ such that $u_{1} r \neq 0$ and $u_{2} r \in D$. Because $R$ is prime we can find $u \in U$ such that $u_{1}(r u) \neq 0$. As $u_{2}(r u) \in D U$, we see that $D U$ is a dense right ideal of $U . q(D U) \subseteq R U \subseteq U$, and so (3) is proved. Finally, suppose $q D=0$ for some dense right ideal $D$ of $U$. By Lemma $1, D U$ is a dense right ideal of $R$. Then $q(D U)=0$ and thus $q=0$, showing (4).

If $e \neq 0,1$ is an idempotent in a ring $R$, we set $e_{1}=e$ and write $R_{11}=e_{1} R e_{1}$, $R_{12}=e_{1} R\left(1-e_{1}\right), R_{21}=\left(1-e_{1}\right) R e_{1}$, and $R_{22}=\left(1-e_{1}\right) R\left(1-e_{1}\right)$. Then $R$ may be written in its Peirce decomposition $R_{11} \oplus R_{12} \oplus R_{21} \oplus R_{22}$. Note that $R$ need not have an identity element.

LeMma 2. Let $R$ be a prime ring containing an idempotent $e \neq 0,1$. Then

(a) If $D$ is a dense right ideal of $e R e, D R+(1-e) R$ is a dense right ideal of $R$.

(b) If $D$ is a dense right ideal of $R, D \cap e R e$ is a dense right ideal of $e R e$.

Proof. Let $r \neq 0, s \in R$, and write $s=s_{11}+s_{12}+s_{22}+s_{21}, s_{i j} \in R_{i j}$. We can find $x, y \in R$ such that $a_{1}=e_{1} x r y e_{1} \neq 0$, since $R$ is prime. Now write sye $e_{1}=t_{11}+t_{12}+t_{21}$ $+t_{22}$. Since $D$ is a dense right ideal of $R_{11}$, there exists $u_{1} \in R_{11}$ such that $a_{1} u_{1} \neq 0$ 
and $t_{11} u_{1} \in D$. From $a_{1} u_{1} \neq 0$ we have $r\left(y e_{1} u_{1}\right) \neq 0$ and $s\left(y e_{1} u_{1}\right)=t_{11} u_{1}+t_{21} u_{1} \in D$ $+(1-e) R$. Thus (a) is proved. To verify (b) let $x_{1} \neq 0, y_{1} \in R_{11}$. There exists $r \in R$ such that $x_{1} r \neq 0$ and $y_{1} r \in D$. There exists $c \in R$ such that $x_{1} r c e_{1} \neq 0$, since $R$ is prime. Then $x_{1}\left(e_{1} r c e_{1}\right)=x_{1} r c e \neq 0$, and $y_{1}\left(e_{1} r c e_{1}\right)=\left(y_{1} r\right) c e_{1} \in D \cap R_{11}$.

LEMmA 3. Let $f \in \operatorname{Hom}_{R_{11}}\left(D, R_{11}\right)$, where $D$ is a dense right ideal of $R_{11}$. Then $f$ can be extended to $g \in \operatorname{Hom}_{R}(E, R)$, where $E=D R+(1-e) R$.

Proof. Noting that $D R=D+D R_{12}$, we may write an element $x \in E$ in the form $x=d+\sum_{\lambda} d^{\lambda} x_{12}^{\lambda}+y, d, d^{\lambda} \in D, x_{12}^{\lambda} \in R_{12}, y \in(1-e) R$. We then define a mapping $g: E \rightarrow R$ by setting $g(x)=f(d)+\sum_{\lambda} f\left(d^{\lambda}\right) x_{12}^{\lambda}$. To see that $g$ is well defined, suppose $d+\sum_{\lambda} d^{\lambda} x_{12}^{\lambda}+y=0$. Thus $d=0, \sum_{\lambda} d^{\lambda} x_{12}^{\lambda}=0$, and $y=0$. We choose $y_{21} \in R_{21}$ and note that $\sum_{\lambda} d^{\lambda}\left(x_{12}^{\lambda} y_{21}\right)=0$. Applying $f$, we have

$$
0=\sum_{\lambda} f\left(d^{\lambda}\right)\left(x_{12}^{\lambda} y_{21}\right)=\left\{\sum_{\lambda} f\left(d^{\lambda}\right) x_{12}^{\lambda}\right\} y_{21}=0 .
$$

In other words, $\left\{\sum_{\lambda} f\left(d^{\lambda}\right) x_{12}^{\lambda}\right\} R e_{1}=0$, and so, by the primeness of $R, \sum_{\lambda} f\left(d^{\lambda}\right) x_{12}^{\lambda}$ $=0 . g$ is thus a well-defined mapping, and clearly it is additive and extends $f$. Now let $r \in R$ and write $r=r_{11}+r_{12}+r_{21}+r_{22}$. For $d \in D$,

$$
\begin{aligned}
g(d r) & =g\left(d r_{11}+d r_{12}\right)=f\left(d r_{11}\right)+f(d) r_{12}=f(d) r_{11}+f(d) r_{12} \\
& =f(d)\left(r_{11}+r_{12}\right)=g(d) r
\end{aligned}
$$

Furthermore,

$$
\begin{aligned}
d\left(d x_{12} r\right) & =g\left\{d x_{12}\left(r_{22}+r_{21}\right)\right\}=d\left\{d\left(x_{12} r_{22}\right)+d\left(x_{12} r_{21}\right)\right\}=f(d) x_{12} r_{22}+f(d) x_{12} r_{21} \\
& =g\left(d x_{12}\right)\left(r_{22}+r_{21}\right)=g\left(d x_{12}\right) r .
\end{aligned}
$$

It follows that $g \in \operatorname{Hom}_{R}(E, R)$.

THEOREM 2. Let $R$ be a prime ring containing an idempotent $e \neq 0,1$. Then

$$
Q(e R e)=e Q(R) e .
$$

Proof. We set $Q=Q(R)$ and note first that $e R e \subseteq e Q e$. Next let $f \in \operatorname{Hom}_{R_{11}}\left(D, R_{11}\right)$, $D$ a dense right ideal of $R_{11}$. By Lemma 3, $f$ may be extended to $g \in \operatorname{Hom}_{R}(E, R)$, where $E=D R+(1-e) R$ is, by Lemma 2(a), a dense right ideal of $R$. Therefore there exists $q \in Q$ such that $q v=g(v)$ for all $v \in E$. In particular, $q d=f(d)$ for all $d \in D$. Hence $e q e d=f(d)$ and (2) is proved. Now let $q \in e Q e \subseteq Q$. There is a dense right ideal $D$ of $R$ such that $q D \subseteq R$. By Lemma 2(b), $E=D \cap e R e$ is a dense right ideal of $e R e$. Since $q E \subseteq R \cap e R e=e R e$, (3) has been shown. Finally, suppose $q \in e Q e$ such that $q D=0$, where $D$ is a dense right ideal of $e R e$. By Lemma 2(a), $E=D R+(1-e) R$ is a dense right ideal of $R$. Then $q E=0$, which forces $q=0$.

LEMMA 4. If $T$ is a ring contained between $R$ and $Q(R)$ and $D$ is a dense right ideal of $T$, then $D \cap R$ is a dense right ideal of $R$.

Proof. Let $r_{1} \neq 0, r_{2} \in R$ and find $t \in T$ such that $r_{1} t \neq 0$ and $r_{2} t \in D$. From (3) and (4) of the definition of $Q(R)$, there exists a dense right ideal $E$ of $R$ such that 
$0 \neq t E \subseteq R$, and hence that $0 \neq\left(r_{1} t\right) E \subseteq R$. Therefore there is an $x \in E$ such that $0 \neq r_{1}(t x)$ and $r_{2}(t x) \in D \cap R$.

THEOREM 3. Let $T$ be a ring contained between $R$ and $Q(R)$. Then $Q(T)=Q(R)$.

Proof. Let $f \in \operatorname{Hom}_{T}(D, T), D$ a dense right ideal of $T$. By Lemma $4, D \cap R$ is a dense right ideal of $R$. Let $E=\{x \in D \cap R \mid f(x) \in R\}$. To see that $E$ is a dense right ideal of $R$, let $r_{1} \neq 0, r_{2} \in R$ and pick $r \in R$ such that $r_{1} r \neq 0$ and $r_{2} r \in D \cap R$. Since $f\left(r_{2} r\right) \in Q$, there is a dense right ideal $F$ of $R$ such that $f\left(r_{2} r\right) F \subseteq R$. Since $r_{1} r \neq 0, r_{1} r F \neq 0$. Hence there is an $x \in F$ such that $r_{1}(r x) \neq 0$. Also $f\left(r_{2} r x\right)=f\left(r_{2} r\right) x \in R$, and so $r_{2}(r x) \in E$. It follows that $f \in \operatorname{Hom}_{R}(E, R)$. Thus there is $q \in Q$ such that $q v=f(v)$ for all $v \in E$. Now let $d \in D$. We know that $G=\{x \in R \mid d x \in E\}$ is a dense right ideal of $R$. In view of this $\{q d-f(d)\} x=q(d x)$ $-f(d x)=0$ for all $x \in G$. Therefore $q d=f(d)$ for all $d \in D$ and (2) has been proved.

Next let $q \in Q . q D \subseteq R$ for some dense right ideal of $R$. Let $t_{1} \neq 0, t_{2} \in T$, and choose $x \in R$ so that $0 \neq t_{1} x \in R$ and $t_{2} x \in R$. There is an $r \in R$ such that $t_{1}(x r) \neq 0$ and $t_{2}(x r) \in D$. Hence $D T$ is a dense right ideal of $T$ and $q(D T) \subseteq R T \subseteq T$, showing (3). Finally, suppose $q D=0$ for some dense right ideal $D$ of $T$. By Lemma 4, $D \cap R$ is a dense right ideal of $R$. Since $q(D \cap R)=0$, we obtain $q=0$. This completes the proof that $Q(T)=Q(R)$.

We conclude this section by remarking that if $R$ is a prime ring, then the three related rings studied in Theorems 1,2 , and 3 are also prime rings. Indeed, it is well known that a nonzero ideal $U$ of $R$ is again a prime ring, and that $e R e$ is a prime ring. To see that $T$ is a prime ring, where $R \subseteq T \subseteq Q$, suppose $a T b=0$, with $a \neq 0, b \neq 0 \in T$. As we have seen before, there is a dense right ideal $D$ of $R$ such that $0 \neq a D \subseteq R$ and $0 \neq b D \subseteq R$. Choose $x, y \in D$ such that $a x \neq 0$ and $b y \neq 0$. Then $(a x) R(b y) \subseteq a T b y=0$, a contradiction.

3. Extending the center of a prime ring. We assume throughout this section that $R$ is a prime ring such that $W(R)=0$, i.e., such that $Q=Q(R)$, the complete ring of right quotients of $R$, exists. $C$ will denote the center of $Q$. We always have $1 \in C$, although $R$ does not necessarily have an identity element. $R_{C}=C+R C$ will denote the subring of $Q$ generated by $R$ and $C$.

LEMMA 5. $C=\{q \in Q \mid q x=x q$ for all $x \in R\}$ and $C$ is a field.

Proof. Suppose $q x=x q$ for all $x \in R$. Let $w \in Q$, and choose a dense right ideal $D$ of $R$ such that $w D \subseteq R$. For $d \in D,(q w) d=q(w d)=(w d) q=w(d q)=q(q d)=(w q) d$. It follows that $q w=w q$, and so $q \in C$.

Next let $0 \neq c \in C$. There exists a dense right ideal $D$ of $R$ such that $0 \neq c D \subseteq R$. Let $r_{1} \neq 0, r_{2} \in R$. There is an $x \in D$ such that $r_{1} x \neq 0$ since $r_{1} \neq 0$. We next find a $y \in R$ such that $r_{1} x y \neq 0$ and $r_{2} x y \in D$. Then $r_{1}(c x y)=\left(r_{1} x y\right) c \neq 0$ (since $c$ is a regular element) and $r_{2}(c x y)=\left(r_{2} x y\right) c \in D c=c D$. We have thus shown that $c D$ is a dense right ideal of $R$. The mapping $f: c D \rightarrow R$ given by $f(c d)=d, d \in D$, is a 
well-defined $R$-homomorphism since $c$ is regular. There is a $q \in Q$ such that $q c d=f(c d)=d$ for all $d \in D$. Therefore $q c=1$, and so $C$ is a field.

COROLlary. Let $R$ be a prime ring with $W(R)=0$. Then $R_{C}$ is a prime ring whose center is the field $C$.

THEOREM 4. Let $R$ be a prime ring with $W(R)=0$, and suppose $a x b=b x a$ for some $a, b \in R$ and for all $x \in R$. Then $a$ and $b$ are $C$-dependent.

Proof. We may assume that $a \neq 0$ and $b \neq 0$. Let $D=R a R$. By the corollary of Lemma $1, D$ is a dense (right) ideal of $R$. We define a mapping $f: D \rightarrow R$ according to the rule

$$
\sum_{i} x_{i} a y_{i} \rightarrow \sum x_{i} b y_{i}, \quad x_{i}, y_{i} \in R
$$

To show that $f$ is well defined, we suppose that $\sum x_{i} a y_{i}=0$. Then $0=b r \sum x_{i} a y_{i}$ $=\sum b\left(r x_{i}\right) a y_{i}$ for all $r \in R$, and thus, by our hypothesis, $\sum a\left(r x_{i}\right) b y_{i}=\operatorname{ar}\left(\sum x_{i} b y_{i}\right)=0$. Since $R$ is prime we conclude that $\sum x_{i} b y_{i}=0$, showing that $f$ is well defined. $f$ is an $R$-homomorphism because

$$
f\{(x a y) r\}=f\{x a(y r)\}=x b(y r)=f(x a y) r
$$

for all $x, y, r \in R$. Now, from (2) of the definition of $Q$, we may find $q \in Q$ such that $q d=f(d)$ for all $d \in D$. If $r \in R$, then $q r(x a y)=q(r x a y)=f(r x a y)=r x b y=r f(x a y)$ $=r q(x a y)$, showing that $(q r-r q) D=0$. Therefore $q r=r q$ for all $r \in R$, and so, by Lemma 5, $q \in C$. In particular, $x(q a) y=q(x a y)=f(x a y)=x b y$ for all $x, y \in R$, i.e., $R(q a-b) R=0$. Since $R$ is prime, we obtain $q a=b$.

We pause at this point to mention an example (communicated orally to us by P. M. Cohn) which shows that $C$ may properly contain $Z$, the center of $R$, even if $Z$ is a field. In fact, let $F$ be a field possessing an automorphism $\sigma$ of infinite period. Let $R$ be the "skew" polynomial ring $F[x, y]$, with the usual addition and with multiplication conforming to the rules $x y=y x, x a=a^{\sigma} x$, and $y a=a^{\sigma} y$, for all $a \in F . R$ is a right and left Noetherian integral domain whose center

$$
Z=\left\{a \in F \mid a^{\sigma}=a\right\} .
$$

One can then show that (in $Q(R)) x y^{-1} \in C$ but $x y^{-1} \notin R$.

THEOREM 5. Let $R$ be a prime ring with $W(R)=0$, and let $T=R_{C}$. Then $T^{\prime} \otimes_{C} T$ $\cong T_{l} T_{r}$, where $T^{\prime}$ is the opposite ring of $T$ and $T_{l}\left(T_{r}\right)$ is the ring of left (right) multiplications of $T$ acting on $T$.

Proof. One checks in a straightforward way that the mapping

$$
\sum_{i} a_{i}^{\prime} \otimes b_{i} \rightarrow \sum_{i} a_{i l} b_{i r}, \quad a_{i}^{\prime} \in T^{\prime}, \quad b_{i} \in T,
$$

is a homomorphism of the ring $T^{\prime} \otimes_{C} T$ onto the ring $T_{l} T_{r}$. Suppose the kernel $K \neq 0$. Choose a nonzero element $\sum_{i=1}^{m} a_{i}^{\prime} \otimes b_{i}$ in $K$ with minimal "length" $m$. 
Then $\left\{a_{1}, \ldots, a_{m}\right\}$ and $\left\{b_{1}, \ldots, b_{m}\right\}$ are both independent sets over $C$. For $x \in T$

$$
\left(a_{m}^{\prime} x^{\prime} \otimes 1\right)\left(\sum_{i=1}^{m} a_{i}^{\prime} \otimes b_{i}\right)-\left(\sum_{i=1}^{m} a_{i}^{\prime} \otimes b_{i}\right)\left(x^{\prime} a_{m}^{\prime} \otimes 1\right)=\sum_{i=1}^{m-1}\left(a_{m}^{\prime} x^{\prime} a_{i}^{\prime}-a_{i}^{\prime} x^{\prime} a_{m}^{\prime}\right) \otimes b_{i}
$$

is an element in $K$ of length less than $m$, and so must be 0 . By the independence of $\left\{b_{i}\right\}$, one sees that

$$
a_{m} x a_{i}-a_{i} x a_{m}=0, \quad i=1,2, \ldots, m,
$$

for all $x \in T$. By Theorem 3, $Q(T)=Q(R)$, and so, by applying Theorem 4 to the $\operatorname{ring} T$, we have $a_{m}=c_{i} a_{i}, c_{i} \in C, i=1,2, \ldots, m-1$. Thus a contradiction is reached, since the $\left\{a_{i}\right\}$ are $C$-independent.

Corollary. Let $R$ be a prime ring with 1 , and let $T=R C$. Suppose $\sum_{i=1}^{n} a_{i} x b_{i}=0$ for all $x \in T$. Then either $\left\{a_{i}\right\}$ are $C$-dependent or $\left\{b_{i}\right\}$ are $C$-dependent.

Proof. Suppose $\left\{a_{i}\right\},\left\{b_{i}\right\}$ are both $C$-independent sets. Since $\sum_{i} a_{i l} b_{i r}=0$, by the theorem $\sum a_{i}^{\prime} \otimes b_{i}=0$, a contradiction.

We only mention this corollary because we feel it may shed some light on the problem of attempting to generalize Amitsur's results on generalized polynomial identities for primitive rings [5] to analogous results for prime rings. Our corollary amounts in effect to a statement that there does not exist a prime ring $R$ with 1 which satisfies a nontrivial "generalized" polynomial identity of degree 1 .

For the remainder of this section we apply Theorem 5 to obtain some needed results on the structure of $R$ as a Lie ring.

THEOREM 6. Let $R$ be a prime ring with $W(R)=0$, of characteristic $\neq 2,3$, and let $a$ be an element of $T=R_{C}$. Then $a=e+c$, e an idempotent in $T, c \in C$, if and only if

$$
[[[x a] a] a]=[x a] \text { for all } x \in R .
$$

(Note that here and frequently throughout the rest of the paper $[x a]$ will stand for $x a-a x$.)

Proof. If $a=e+c$, (1) is straightforward to verify. Conversely, if (1) holds, it is easy to first check that

$$
[[[x a] a] a]=[x a] \text { for all } x \in T \text {. }
$$

(2) may be written as

$$
a_{r}^{3}-3 a_{l} a_{r}^{2}+3 a_{r} a_{l}^{2}-a_{l}^{3}=a_{r}-a_{l} .
$$

Since $T_{l} T_{r} \cong T^{\prime} \otimes_{C} T$ by Theorem 5 , we may replace (3) by

$$
1 \otimes a^{3}-3 a \otimes a^{2}+\left(3 a^{2}-1\right) \otimes a+\left(a-a^{3}\right) \otimes 1=0 .
$$

Therefore $\left\{1, a, a^{2}, a^{3}\right\}$ is a $C$-dependent set. If $a \in C$, we are already finished, so we may assume that $\{1, a\}$ is an independent set. If $\left\{1, a, a^{2}\right\}$ is an independent set, 
then $a^{3}=\alpha a^{2}+\beta a+\gamma, \alpha, \beta, \gamma \in C$. Substituting this in (4) yields

$$
1 \otimes\left\{\alpha a^{2}+(\beta-1) a\right\}+a \otimes\left\{-3 a^{2}+(1-\beta) a\right\}+a^{2} \otimes(3 a-\alpha)=0 .
$$

In particular, $3 a=\alpha$, a contradiction since the characteristic of $T$ is different from 3 . We are thus forced to assume

$$
a^{2}=\alpha a+\beta
$$

whence

$$
a^{3}=\left(\alpha^{2}+\beta\right) a+\alpha \beta .
$$

Substitution of (5) and (6) in (4) yields

$$
1 \otimes\left(\alpha^{\rho}+4 \beta-1\right) a+a \otimes\left(1-\alpha^{2}-4 \beta\right)=0
$$

or

$$
\alpha^{2}+4 \beta-1=0
$$

Using (5) and (7) one verifies directly that $e=a+\frac{1}{2}(1-\alpha)$ is an idempotent, and thus $a=e+c$, where $c=-\frac{1}{2}(1-\alpha)$.

The next three lemmas are due to Herstein.

LEMMA 6. Let $R$ be a prime ring of characteristic $\neq 2$, let $I$ be a nonzero ideal of $R$, and suppose $[[x a] a]=0$ for all $x \in I$. Then a lies in the center of $R$.

Proof (Herstein). From $[[x y, a] a]=0$ and $[x y, a]=x[y, a]+[x, a] y, x, y \in I$, and char $R \neq 2$, one obtains $[x, a][y, a]=0$. Setting $y=r x, x, r \in I$, one sees that $[x, a] r[x, a]=0$, i.e., $[x, a] I[x, a]=0$. By the primeness of $R,[x, a]=0$ for all $x \in I$. Replacing $x \in I$ by $x r \in I, r \in R$, we then have $x($ ra-ar $)=0$ for all $x \in I$, which forces $r a-a r=0$ for all $r \in R$.

LeMma 7. Let $R$ be a prime ring such that $[[R, R],[R, R]]=0$. Then $R$ is commutative.

Proof. If $a \notin Z$ (the center of $R$ ), then by Lemma 6 there is an $x \in R$ such that $b=[x, a] \notin Z$. But by hypothesis $[[y, b] b]=0$ for all $y \in R$, a contradiction to Lemma 6.

LEMMA 8. Let $R$ be a prime ring of characteristic $\neq 2$, and let $U$ be a Lie ideal of $R$. Then either $U \subseteq Z$ or $U \supseteq[I, R]$ for some nonzero ideal $I$ of $R$.

Proof. Let $T(U)=\{x \in R \mid[x, R] \subseteq U\} . T(U)$ is easily seen to be a Lie ideal of $R$. Furthermore, letting $a, b \in T(U), r \in R$, and writing

$$
(a b) r-r(a b)=a(b r)-(b r) a+b(r a)-(r a) b,
$$

one sees that $T(U)$ is also an associative subring of $R$. By [8, p. 9, Lemma 2], either $T(U) \subseteq Z$ or $T(U) \supseteq I$ for some ideal $I$ of $R$. If $T(U) \subseteq Z$, then $U \subseteq T(U) \subseteq Z$. If $T(U) \supseteq I$, then $U \supseteq[T(U), R] \supseteq[I, R]$. 
LeMma 9. Let $R$ be a prime ring with 1 and let $a$ and $b$ be idempotents of $T=R_{C}(=R C)$ such that $a b=b a$ and

$$
[[[[x a] b] a] b]+[[x a] b]=0 \text { for all } x \in R .
$$

Then either $a b=0$ or $(1-a)(1-b)=0$.

Proof. (1) holds in the prime ring $T$. Multiplication of (1) on the left by $a b$ then gives us

$$
a b x a b-a b x b-a b x a+a b x=0, \quad x \in T .
$$

Factoring, we have $a b T(1-a)(1-b)=0$, from which one concludes that either $a b=0$ or $(1-a)(1-b)=0$.

LEMMA 10. Let $R$ be a prime ring with $W(R)=0$, and let $e \neq 0,1$ be an idempotent in $T=T_{C}$. Then the center of eTe is $e C$.

Proof. Suppose $(e a e)($ exe $)=($ exe $)(e a e)$ for all $x \in R$. Setting $b=e a e$, we have $b x e=e x b$ for all $x \in R$, and indeed for all $x \in T$. We then apply Theorem 4 directly to the ring $T$ to conclude that $b=c e$, for some $c \in C$.

4. Lie isomorphisms of prime rings: Case 1a. Henceforth in this paper we shall assume that $S$ is a prime ring with 1 of characteristic different from 2 and 3 , and containing two nonzero orthogonal idempotents $e_{1}$ and $e_{2}$ whose sum is 1 . We assume further that there exists a Lie isomorphism $\phi$ of $S$ onto a prime ring $R$ with 1 . We remark that $\phi(x) \in Z$, the center of $R$, if and only if $x \in Y$, the center of $S . R$ will be regarded as a subring of $Q=Q(R)$, its complete ring of right quotients, and we will set $T=R C$, where $C$ is the center of $Q$. The results of $\S \S 1$ and 2 will be at our disposal.

THEOREM 7. Either

(Case 1)

$$
\phi\left(e_{i}\right)=c_{i}+f_{i}, \quad i=1,2,
$$

or

(Case 2)

$$
\phi\left(e_{i}\right)=c_{i}-f_{i}, \quad i=1,2,
$$

where $c_{i} \in C$, and $f_{1}, f_{2}$ are orthogonal idempotents of $T$ whose sum is 1 .

Proof. Set $\phi\left(e_{i}\right)=g_{i}$. Since $\left[\left[\left[x e_{i}\right] e_{i}\right] e_{i}\right]=\left[x e_{i}\right]$ for all $x \in S$ and $\phi$ is onto, it follows that $\left[\left[\left[y g_{i}\right] g_{i}\right] g_{i}\right]=\left[y g_{i}\right]$ for all $y \in R$. By Theorem $6 g_{i}=c_{i}+f_{i}, f_{i}$ an idempotent in $T=R C, c_{i} \in C, i=1,2$. A direct calculation shows that

$$
\left[\left[\left[\left[x e_{1}\right] e_{2}\right] e_{1}\right] e_{2}\right]+\left[\left[x e_{1}\right] e_{2}\right]=0
$$

for all $x \in S$. Since $c_{i} \in C$ we then have

$$
\left[\left[\left[\left[y f_{1}\right] f_{2}\right] f_{1}\right] f_{2}\right]+\left[\left[y f_{1}\right] f_{2}\right]=0
$$

for all $y \in R$. Also, from $\left[e_{1}, e_{2}\right]=0$, we see that $\left[f_{1}, f_{2}\right]=0$. Lemma 9 then says 
that either $f_{1} f_{2}=0$ or $\left(1-f_{1}\right)\left(1-f_{2}\right)=0$. Therefore either $\phi\left(e_{i}\right)=c_{i}+f_{i},\left\{f_{i}\right\}$ orthogonal idempotents, $i=1,2$, or $\phi\left(e_{i}\right)=c_{i}+f_{i}=\left(c_{i}+1\right)-\left(1-f_{i}\right)=b_{i}-h_{i}, b_{\imath} \in C,\left\{h_{i}\right\}$ orthogonal idempotents, $i=1,2$. Finally, from $0=[x, 1]=\left[x, e_{1}+e_{2}\right]$ for all $x \in S$, we see that $\left[y, f_{1}+f_{2}\right]=0$ for all $y \in R$. Therefore the idempotent $f_{1}+f_{2}$ lies in $C$ and must be equal to 1 .

Until further notice in this section we assume that Case 1 holds, i.e., $\phi\left(e_{i}\right)=c_{i}+f_{i}$, $i=1,2$. We also recall the notation $S_{i j}=e_{i} S e_{j}$ and $T_{i j}=f_{i} T f_{j}$. Clearly, $S=\oplus \sum_{i, j=1}^{2} S_{i j}$, and, in view of Theorem $7, T=\oplus \sum_{i, j=1}^{2} T_{i j}$.

LEMMA 11. $\phi\left(S_{i j}\right) \subseteq T_{i j}, i \neq j$.

Proof. Let $x \in S_{12}$ and set $y=\phi(x)$. Since $x=\left[e_{1}\left[x e_{2}\right]\right]$ and $x=\left[e_{1} x\right]$ we have

$$
\begin{aligned}
y & =\left[f_{1}\left[y f_{2}\right]\right]=f_{1} y f_{2}+f_{2} y f_{1} \\
& =f_{1}\left(f_{1} y-y f_{1}\right) f_{2}+f_{2}\left(f_{1} y-y f_{1}\right) f_{1}=f_{1} y f_{2}-f_{2} y f_{1} .
\end{aligned}
$$

Therefore $y=f_{1} y f_{2} \in T_{12}$, since char $T \neq 2$.

LEMMA 12. $\phi\left(S_{i i}\right) \subseteq T_{11}+T_{22}$.

Proof. Let $x_{1} \in S_{11}$ and set $\phi\left(x_{1}\right)=\sum_{i, j=1}^{2} y_{i j}=y, y_{i j} \in T_{i j}$. From $\left[x_{1} e_{2}\right]=0$ we conclude that $\left[y f_{2}\right]=y_{12}-y_{21}=0$. Hence $y_{12}=0=y_{21}$, i.e., $\phi\left(x_{1}\right) \in T_{11}+T_{22}$.

LEMMA 13. $T_{i j}=\phi\left(S_{i j}\right) C, \quad i \neq j$.

Proof. We first note that $T_{12}=f_{1} R C f_{2}=\left(f_{1} R f_{2}\right) C$, and so we consider an element $f_{1} \phi(s) f_{2} c, c \in C, s \in S$. Writing $s=\left(s_{11}+s_{22}\right)+s_{12}+s_{21}, s_{i j} \in S_{i j}$, we have

$$
\phi(s)=\phi\left(s_{11}+s_{22}\right)+\phi\left(s_{12}\right)+\phi\left(s_{21}\right) .
$$

By Lemmas 11 and 12, this yields $f_{1} \phi(s) f_{2} c=f_{1} \phi\left(s_{12}\right) f_{2} c=\phi\left(s_{12}\right) c \in \phi\left(S_{12}\right) C$.

Now consider the following sets:

$$
\begin{aligned}
A_{1}=\left\{a \in S_{11} \mid \phi(a) \in T_{11}+C\right\}, & A_{2}=\left\{a \in S_{11} \mid \phi(a) \in T_{22}+C\right\}, \\
B_{1}=\left\{b \in S_{22} \mid \phi(b) \in T_{11}+C\right\}, & B_{2}=\left\{b \in S_{22} \mid \phi(b) \in T_{22}+C\right\} .
\end{aligned}
$$

LEMMA 14. $A_{1}=S_{11}$ or $A_{2}=S_{11}$, and $B_{1}=S_{22}$ or $B_{2}=S_{22}$.

Proof. Suppose $A_{1} \neq S_{11}$ and $A_{2} \neq S_{11}$. Then there exists $x_{1} \in S_{11}$ such that $x_{1} \notin A_{1}$, i.e., $\phi\left(x_{1}\right)=u_{1}+u_{2}, u_{1} \in T_{11}, u_{2} \in T_{22}, u_{2} \notin f_{2} C$. Since $T$ is a prime ring, $I=T_{21} T_{12}$ is a nonzero ideal of the prime ring $T_{22}$. Furthermore, we see from Lemma 13 that $I=\phi\left(S_{21}\right) \phi\left(S_{12}\right) C$. By Lemma 10, the center of $T_{22}$ is $f_{2} C$. Applying Lemma 6 to the ring $T_{22}$ and the ideal $I$, we can find $w_{2} \in I$ such that $u_{2} w_{2}$ $w_{2} u_{2} \notin f_{2} C$. Since $I=\phi\left(S_{21}\right) \phi\left(S_{12}\right) C$ there exists $v_{2}=\phi\left(x_{21}\right) \phi\left(x_{12}\right) \in I$ such that $u_{2} v_{2}-v_{2} u_{2} \notin f_{2} C$. By Lemmas 11 and 12 , we may write $v_{2}=\phi\left(a_{1}+a_{2}\right)$, where $a_{i} \in S_{i i}$. Set $y_{1}=x_{1} a_{1}-a_{1} x_{1}$. Then

$$
\begin{aligned}
\phi\left(y_{1}\right) & =\phi\left\{x_{1}\left(a_{1}+a_{2}\right)-\left(a_{1}+a_{2}\right) x_{1}\right\}=\left(u_{1}+u_{2}\right) v_{2}-v_{2}\left(u_{1}+u_{2}\right) \\
& =u_{2} v_{2}-v_{2} u_{2} \notin f_{2} C .
\end{aligned}
$$


Now let $U=\left\{x \in S_{11} \mid \phi(x) \in T_{22}\right\}$. If $x \in U$ and $r \in S_{11}$, then

$$
\phi(x r-r x)=\phi(x) \phi(r)-\phi(r) \phi(x) \in T_{22},
$$

by Lemma 12. Therefore $U$ is a Lie ideal of $S_{11}$. We have already shown that $y_{1} \in U$. Since $\phi\left(y_{1}\right) \notin f_{2} C$, there exists $w_{2} \in T_{22}$ such that $\left[\phi\left(y_{1}\right), w_{2}\right] \neq 0$. Because $w_{2} \in T=R C$ we may write

$$
w_{2}=\sum_{\lambda} \phi\left(s^{\lambda}\right) c_{\lambda}=\sum_{\lambda}\left\{\phi\left(s_{11}^{\lambda}+s_{22}^{\lambda}\right)+\phi\left(s_{12}^{\lambda}\right)+\phi\left(s_{21}^{\lambda}\right)\right\} c_{\lambda}, \quad s_{i j}^{\lambda} \in S_{i j}, \quad c_{\lambda} \in C .
$$

By Lemmas 11 and 12, it follows that $w_{2}=\sum_{\lambda} \phi\left(s_{11}^{\lambda}+s_{22}^{\lambda}\right) c_{\lambda}$, and so there exist $s_{11} \in S_{11}, s_{22} \in S_{22}$ such that $0 \neq\left[\phi\left(y_{1}\right), \phi\left(s_{11} s_{22}\right)\right]=\phi\left(y_{1} s_{11}-s_{11} y_{1}\right)$. This shows that $y_{1} \notin Z_{1}$, the center of $S_{11}$. Hence by Lemma 8 , there is a nonzero ideal $J$ of $S_{11}$ for which $U \supseteq\left[J, S_{11}\right]$. Similarly $V=\left\{x \in S_{11} \mid \phi(x) \in T_{11}\right\}$ is a Lie ideal of $S_{11}$ containing $\left[K, S_{11}\right]$ for some nonzero ideal $K$ of $S_{11} . L=J \cap K$ is again a nonzero ideal of the prime ring $S_{11}$. Thus $U \supseteq\left[L, S_{11}\right]$ and $V \supseteq\left[L, S_{11}\right]$. Let $u \in U$ and $v \in V$. Then $\phi(u v-v u)=\phi(u) \phi(v)-\phi(v) \phi(u)=0$, i.e., $u v-v u=0$, or $[U, V]=0$. Thus $\left[\left[L, S_{11}\right],\left[L, S_{11}\right]\right]=0$ and in particular $[[L, L],[L, L]]=0$. Since $L$ is itself a prime ring, $L$ is commutative by Lemma 7 . But it is well known that if a nonzero ideal of a prime ring is commutative then the ring itself is commutative. Therefore $S_{11}$ is commutative, and we have a contradiction to the fact that $y_{1}=x_{1} a_{1}-a_{1} x_{1} \neq 0$.

\section{THEOREM 8. Either}

(Case 1a)

$$
A_{1}=S_{11} \text { and } \quad B_{2}=S_{22}
$$

or

(Case 1b)

$$
A_{2}=S_{11} \quad \text { and } \quad B_{1}=S_{22}
$$

Proof. Suppose that neither of these two cases prevails. We may assume then, in view of Lemma 14 , that $A_{1}=S_{11}$ and $B_{1}=S_{22}$. If $\phi\left(S_{11}\right) \subseteq f_{1} C+f_{2} C$, then $A_{2}=S_{11}$ and Case $1 \mathrm{~b}$ would hold. If $\phi\left(S_{22}\right) \subseteq f_{1} C+f_{2} C$, then $B_{2}=S_{22}$ and Case 1a would hold. Therefore we may assume that there is an $x_{1} \in S_{11}$ such that $\phi\left(x_{1}\right)$ $=u_{1}+c_{2}, u_{1} \in T_{11}, u_{1} \notin f_{1} C, c_{2} \in f_{2} C$, and that there is an $x_{2} \in S_{22}$ such that $\phi\left(x_{2}\right)=v_{1}+d_{2}, v_{1} \in T_{11}, v_{1} \notin f_{1} C, d_{2} \in f_{2} C$. As we have seen before, $\phi\left(S_{12}\right) \phi\left(S_{21}\right) C$ is a nonzero ideal of the prime ring $T_{11}$. Hence by Lemma 6 there exists

$$
t_{1} \in \phi\left(S_{12}\right) \phi\left(S_{21}\right)
$$

such that $\left[\left[t_{1} u_{1}\right] u_{1}\right] \neq 0$. Since $t_{1} \in R$, we may write, using Lemmas 11 and 12 , $t_{1}=\phi\left(b_{1}+b_{2}\right), b_{i} \in S_{i i}$. Setting $y_{1}=x_{1} b_{1}-b_{1} x_{1} \in S_{11}$, we see that

$$
\begin{aligned}
\phi\left(y_{1}\right) & =\phi\left\{x_{1}\left(b_{1}+b_{2}\right)-\left(b_{1}+b_{2}\right) x_{1}\right\}=\left(u_{1}+c_{2}\right) t_{1}-t_{1}\left(u_{1}+c_{2}\right) \\
& =u_{1} t_{1}-t_{1} u_{1} \notin f_{1} C .
\end{aligned}
$$

We claim that the additive subgroups $U$ of $T_{11}$ spanned by all elements of the form $\phi\left(s_{1}\right) c, s_{1} \in S_{11}, \phi\left(s_{1}\right) \in T_{11}, c \in C$, is a Lie ideal of $T_{11}$. Indeed, if $t \in T_{11}$, we 
may write $t=\sum_{\lambda} \phi\left(w^{\lambda}\right) c_{\lambda}=\sum \phi\left(w_{1}^{\lambda}+w_{2}^{\lambda}\right) c_{\lambda}, c_{\lambda} \in C, w_{i}^{\lambda} \in S_{i i}$, making use of Lemmas 11 and 12. In view of this it suffices to note that

$$
\left[\phi\left(x_{1}\right) c, \phi\left(w_{1}+w_{2}\right) d\right]=\phi\left[s_{1}, w_{1}\right](c d) \in U, \quad c, d \in C .
$$

Since $\phi\left(y_{1}\right) \in U$ there exists, by Lemma 8 , an ideal $I$ of $T_{11}$ such that $U \supseteq\left[I, T_{11}\right]$. Similarly, the additive subgroup $V$ of $T_{11}$ spanned by all elements of the form $\phi\left(s_{2}\right) c, s_{2} \in S_{22}, \phi\left(s_{2}\right) \in T_{11}, c \in C$, is a Lie ideal of $T_{11}$ not contained in the center $f_{1} C$ of $T_{11}$. So again by Lemma $8, V \supseteq\left[J, T_{11}\right]$ for some nonzero ideal $J$ of $T_{11}$. $K=I \cap J$ is a nonzero ideal of the prime ring $T_{11}$, and thus $U \supseteq\left[K, T_{11}\right]$ and $V \supseteq\left[K, T_{11}\right]$. But $[U, V]=0$ since $\left[\phi\left(s_{1}\right) c, \phi\left(s_{2}\right) d\right]=\phi\left[s_{1}, s_{2}\right](c d)=0, s_{i} \in S_{i i}, \phi\left(s_{i}\right) \in T_{i i}$, $c, d \in C, i=1,2$. This in turn forces $[[K, K],[K, K]]=0$, a contradiction to Lemma 7.

For the remainder of this section we shall assume that Case 1a holds. Lemma 11 and Theorem 8 show us that

(A) if $x \in S_{i j}, i \neq j$, then $\phi(x)=x^{*} \in T_{i j}$,

(B) if $x \in S_{i i}$, then $\phi(x)=x^{*}+c, x^{*} \in T_{i i}, c \in C$.

We note that in (B) $x^{*}$ and $c$ are uniquely determined. Indeed, if $\phi(x)=x^{*}+c$ $=y+d, y \in T_{i i}, d \in C$, then $x^{*}-y \in C \cap T_{i i}=0$. Hence $y=x^{*}$ and $c=d$.

Relations (A) and (B) enable us to define in a natural way a mapping $\sigma$ of $S$ into $T$ according to the rule $\sigma(x)=x^{*}, x \in S_{i j}, i, j=1,2$. A mapping $\tau$ of $S$ into $C$ is then defined by $\tau(x)=\phi(x)-\sigma(x)$.

LEMMA 15. $\sigma$ (and hence $\tau$ ) is additive.

Proof. It suffices to show that $\sigma$ is additive on $S_{i i}$. Let $x$ and $y$ be elements of $S_{i i}$. Then

$$
\begin{aligned}
\sigma(x+y)-\sigma(x)-\sigma(y) & =\phi(x+y)-\tau(x+y)-\phi(x)+\tau(x)-\phi(y)+\tau(y) \\
& =\tau(x)+\tau(y)-\tau(x+y) \in C \cap T_{i i}=0 .
\end{aligned}
$$

Thus $\sigma(x+y)=\sigma(x)+\sigma(y)$.

LemMA 16. Let $x \in S_{i i}$ and $y \in S_{i j}, i \neq j$. Then $\sigma(x y)=\sigma(x) \sigma(y)$.

Proof. Let $x_{1} \in S_{11}$ and let $x_{12} \in S_{12}$. Then

$$
\begin{aligned}
\sigma\left(x_{1} x_{12}\right)= & \phi\left(x_{1} x_{12}\right)=\phi\left(x_{1} x_{12}-x_{12} x_{1}\right)=\phi\left(x_{1}\right) \phi\left(x_{12}\right)-\phi\left(x_{12}\right) \phi\left(x_{1}\right) \\
& =\sigma\left(x_{1}\right) \sigma\left(x_{12}\right)-\sigma\left(x_{12}\right) \sigma\left(x_{1}\right)=\sigma\left(x_{1}\right) \sigma\left(x_{12}\right) .
\end{aligned}
$$

Lemma 17. Let $x \in S_{i j}$ and let $y \in S_{j i}, i \neq j$. Then $\sigma(x y)=\sigma(x) \sigma(y)$.

Proof. Let $x_{12} \in S_{12}, a_{21} \in S_{21}$, and $s_{12} \in S_{12}$. We apply $\phi$ to $x_{12} a_{21} s_{12}+s_{12} a_{21} x_{12}$ $=\left[\left[x_{12}, a_{21}\right], s_{12}\right]$ and obtain

$$
\phi\left(x_{12} a_{21} s_{12}+s_{12} a_{21} x_{12}\right)=\phi\left(x_{12}\right) \phi\left(a_{21}\right) \phi\left(s_{12}\right)+\phi\left(s_{12}\right) \phi\left(a_{21}\right) \phi\left(x_{12}\right) .
$$


By Lemma 16, (1) becomes

$$
\sigma\left(x_{12} a_{21}\right) \sigma\left(s_{12}\right)+\sigma\left(s_{12}\right) \sigma\left(a_{21} x_{12}\right)=\sigma\left(x_{12}\right) \sigma\left(a_{21}\right) \sigma\left(s_{12}\right)+\sigma\left(s_{12}\right) \sigma\left(a_{21}\right) \sigma\left(x_{12}\right)
$$

or

$$
\left\{\sigma\left(x_{12} a_{21}\right)-\sigma\left(x_{12}\right) \sigma\left(a_{21}\right)\right\} \sigma\left(s_{12}\right)=\sigma\left(s_{12}\right)\left\{\sigma\left(a_{21}\right) \sigma\left(x_{12}\right)-\sigma\left(a_{21} x_{12}\right)\right\} .
$$

An analogous argument shows that, if $s_{21} \in S_{21}$, then

$$
\sigma\left(s_{21}\right)\left\{\sigma\left(x_{12} a_{21}\right)-\sigma\left(x_{12}\right) \sigma\left(a_{21}\right)\right\}=\left\{\sigma\left(a_{21}\right) \sigma\left(x_{12}\right)-\sigma\left(a_{21}\right) \sigma\left(x_{12}\right)\right\} \sigma\left(s_{21}\right) .
$$

The subring $U$ generated by $T_{12}$ and $T_{21}$ is an ideal of $T$ and we know by Lemma 13 that $T_{i j}=\phi\left(S_{i j}\right) C, i \neq j$. Therefore (2) and (3) say that the element

$$
t=\sigma\left(x_{12} a_{21}\right)-\sigma\left(x_{12}\right) \sigma\left(a_{21}\right)+\sigma\left(a_{21}\right) \sigma\left(x_{12}\right)-\sigma\left(a_{21} x_{12}\right)
$$

commutes with every element of $U$. By Lemma 6 we have $t \in C$. Multiplication of (4) on the right by $\sigma\left(x_{12}\right)$ yields

$$
\sigma\left(x_{12} a_{21}\right) \sigma\left(x_{12}\right)-\sigma\left(x_{12}\right) \sigma\left(a_{21}\right) \sigma\left(x_{12}\right)=t \sigma\left(x_{12}\right) .
$$

By Lemma 16, (5) becomes

$$
\sigma\left(x_{12} a_{21} x_{12}\right)-\sigma\left(x_{12}\right) \sigma\left(a_{21}\right) \sigma\left(x_{12}\right)=t \sigma\left(x_{12}\right) .
$$

On the other hand, setting $s_{12}=x_{12}$ in (1) gives $\sigma\left(x_{12} a_{21} x_{12}\right)=\sigma\left(x_{12}\right) \sigma\left(a_{21}\right) \sigma\left(x_{12}\right)$. Therefore (6) becomes $0=t \sigma\left(x_{12}\right)$, whence $t=0$. But then we have in particular from (4) that $\sigma\left(x_{12} a_{21}\right)=\sigma\left(x_{12}\right) \sigma\left(a_{21}\right)$.

Lemma 18. Let $x, y \in S_{i i}$. Then $\sigma(x y)=\sigma(x) \sigma(y)$.

Proof. Let $x_{1}, y_{1} \in S_{11}$ and let $s_{12} \in S_{12}$. Then, using Lemma 16,

$$
\sigma\left(x_{1} y_{1}\right) \sigma\left(s_{12}\right)=\sigma\left(x_{1} y_{1} s_{12}\right)=\sigma\left(x_{1}\right) \sigma\left(y_{1} s_{12}\right)=\sigma\left(x_{1}\right) \sigma\left(y_{1}\right) \sigma\left(s_{12}\right) .
$$

In particular, $\sigma\left(x_{1} y_{1}\right)-\sigma\left(x_{1}\right) \sigma\left(y_{1}\right)$ commutes with $\sigma\left(s_{12}\right)$. Similarly, $\sigma\left(x_{1} y_{1}\right)-$ $\sigma\left(x_{1}\right) \sigma\left(y_{1}\right)$ commutes with all the elements of $\phi\left(S_{21}\right)$. Since $T_{i j}=\phi\left(S_{i j}\right) C, i \neq j$, and the subring generated by $T_{12}$ and $T_{21}$ is an ideal of $T$, we see by Lemma 6 that $\sigma\left(x_{1} y_{1}\right)-\sigma\left(x_{1}\right) \sigma\left(y_{1}\right) \in C \cap T_{11}=0$. Thus $\sigma\left(x_{1} y_{1}\right)=\sigma\left(x_{1}\right) \sigma\left(y_{1}\right)$.

We come now to the main theorem of this paper for Case $1 \mathrm{a}$.

THEOREM 9. For Case 1a, $\sigma$ is an isomorphism of $S$ into $T$.

Proof. From Lemmas 15, 16, 17, and 18, we know that $\sigma$ is a homomorphism of $S$ into $T$. Suppose for some $x=x_{1}+x_{2}+x_{12}+x_{21} \in S$ that $\sigma(x)=0$. From the definition of $\sigma, x_{12}=0=x_{21}$, and so $\sigma\left(x_{1}+x_{2}\right)=0$. From this $\sigma\left(x_{1}+x_{2}\right) \sigma\left(e_{1}\right)=\sigma\left(x_{1}\right)$ $=0$, i.e., $\phi\left(x_{1}\right)=\tau\left(x_{1}\right) \in C$. Therefore $x_{1} \in Y$, the center of $S$, which forces $x_{1}=0$. By the same token $x_{2}=0$, and thus we have shown that $\sigma$ is a one-one mapping.

COROllary. $\tau$ is an additive mapping of $S$ into $C$ such that $\tau(x y-y x)=0$ for all $x, y \in R$. 
5. The main theorem: general case. In this final section we continue with the assumptions made at the beginning of $\S 3$, but now superimpose until further notice the condition that Case $1 \mathrm{~b}$ holds. Our immediate goal is thus to prove the analogue of Theorem 9. Lemma 11 and Theorem 8 combine to yield

(A) if $x \in S_{i j}, i \neq j$, then $\phi(x)=x^{*} \in S_{i j}$,

(B) if $x \in S_{11}$, then $\phi(x)=x^{*}+c, x^{*} \in T_{22}, c \in C$,

(C) if $x \in S_{22}$, then $\phi(x)=x^{*}+c, x^{*} \in T_{11}, c \in C$.

It is again clear that $x^{*}$ and $c$ are uniquely determined and that an additive mapping $\sigma$ of $S$ into $T$ can be defined according to $\sigma(x)=x^{*}, x \in S_{i j}, i, j=1,2$. A mapping $\tau$ of $S$ into $C$ is then defined by $\tau(x)=\phi(x)-\sigma(x), x \in S$.

Lemma 19. Let $x \in S_{i i}$ and let $y \in S_{i j}, i \neq j$. Then $\sigma(x y)=-\sigma(y) \sigma(x)$.

Proof. Let $x_{1} \in S_{11}$ and let $x_{12} \in S_{12}$. Then

$$
\begin{aligned}
\sigma\left(x_{1} x_{12}\right) & =\phi\left(x_{1} x_{12}\right)=\phi\left(x_{1}\right) \phi\left(x_{12}\right)-\phi\left(x_{12}\right) \phi\left(x_{1}\right) \\
& =\sigma\left(x_{1}\right) \sigma\left(x_{12}\right)-\sigma\left(x_{12}\right) \sigma\left(x_{1}\right)=-\sigma\left(x_{12}\right) \sigma\left(x_{1}\right),
\end{aligned}
$$

since $\sigma\left(x_{1}\right) \in T_{22}$.

Lemma 20. Let $x \in S_{i j}$ and let $y \in S_{j i}, i \neq j$. Then $\sigma(x y)=-\sigma(y) \sigma(x)$.

Proof. Let $x_{12} \in S_{12}, a_{21} \in S_{21}$, and $s_{12} \in S_{12}$. As in the proof of Lemma 17, we first obtain

$$
\sigma\left(x_{12} a_{21} s_{12}+s_{12} a_{21} x_{12}\right)=\sigma\left(x_{12}\right) \sigma\left(a_{21}\right) \sigma\left(s_{12}\right)+\sigma\left(s_{12}\right) \sigma\left(a_{21}\right) \sigma\left(x_{12}\right) .
$$

By Lemma 19, (1) becomes

$$
-\sigma\left(s_{12}\right) \sigma\left(x_{12} a_{21}\right)-\sigma\left(a_{21} x_{12}\right) \sigma\left(s_{12}\right)=\sigma\left(x_{12}\right) \sigma\left(a_{21}\right) \sigma\left(s_{12}\right)+\sigma\left(s_{12}\right) \sigma\left(a_{21}\right) \sigma\left(x_{12}\right)
$$

or

(2) $\sigma\left(s_{12}\right)\left\{\sigma\left(a_{21}\right) \sigma\left(x_{12}\right)+\sigma\left(x_{12} a_{21}\right)\right\}=-\left\{\sigma\left(x_{12}\right) \sigma\left(a_{21}\right)+\sigma\left(a_{21}\right) \sigma\left(x_{12}\right)\right\} \sigma\left(s_{12}\right)$.

A similar argument shows that, if $s_{21} \in S_{21}$, then

$$
\sigma\left(s_{21}\right)\left\{\sigma\left(x_{12}\right) \sigma\left(a_{21}\right)+\sigma\left(a_{21} x_{12}\right)\right\}=-\left\{\sigma\left(a_{21}\right) \sigma\left(x_{12}\right)+\sigma\left(a_{12} a_{21}\right)\right\} \sigma\left(s_{21}\right) .
$$

Continuing as in the proof of Lemma 17, we are able to conclude from (2) and (3) that

$$
\sigma\left(x_{12} a_{21}\right)+\sigma\left(a_{21}\right) \sigma\left(x_{12}\right)-\sigma\left(a_{21} x_{12}\right)-\sigma\left(x_{12}\right) \sigma\left(a_{21}\right)
$$

is an element of $C$. From this, again as in the proof of Lemma 17, we finally obtain

$$
\sigma\left(x_{12} a_{21}\right)=-\sigma\left(a_{21}\right) \sigma\left(x_{12}\right) .
$$

We state without proof the analogue of Lemma 18.

LEMMA 21. Let $x, y \in S_{i i}$. Then $\sigma(x y)=-\sigma(y) \sigma(x)$. 
TheOREM 10. For Case 1b, $\sigma$ is the negative of an anti-isomorphism of $S$ into $T$.

Proof. Lemmas 19, 20, and 21 combine to show that $\sigma$ is the negative of a homomorphism $\psi$ of $S$ into $T$, where $\psi(x)=-\sigma(x)$, all $x \in S$. The same reasoning as in the proof of Theorem 9 is used to show that $\psi$ is a one-one mapping of $S$ into $T$.

COROLlaRY. $\tau$ is an additive mapping of $S$ into $T$ such that $\tau(x y-y x)=0$ for all $x, y \in S$.

Having disposed of Case 1 completely, we assume now that Case 2 holds, that is, $\phi\left(e_{i}\right)=c_{i}-f_{i}, i=1,2$ (see Theorem 7). Let $\theta: x \rightarrow x^{\prime}$ be an anti-isomorphism of $R$ onto a ring $R^{\prime}$ (such exists). Then $-\theta$ is easily seen to be a Lie isomorphism of $R$ onto $R^{\prime}$, whence $\psi=-\theta \phi$ is a Lie isomorphism of $S$ onto $R^{\prime}$. Let $P$ be the complete ring of right quotients of $R^{\prime}$, let $B$ be the center of $P$, and set $U=R^{\prime} B$. Our aim is to show that Case 1 arguments apply to $\psi$, so that we may apply Theorems 9 and 10. In order to do this we require the following lemma.

LeMma 22. $\theta$ may be extended to an anti-isomorphism $\chi$ of $T$ onto $U$.

Proof. We first show that $C$ is isomorphic to $B$. Let $c \in C$, and let

$$
D=\{x \in R \mid c x \in R\} .
$$

The image $D^{\prime}$ of $D$ under $\theta$ is an ideal of $R^{\prime}$. Define $g\left(d^{\prime}\right)=(c d)^{\prime}$. Since $g\left(d^{\prime} r^{\prime}\right)$ $=g\left((r d)^{\prime}\right)=(c(r d))^{\prime}=(r(c d))^{\prime}=(c d)^{\prime} r^{\prime}=g\left(d^{\prime}\right) r^{\prime}, r^{\prime} \in R^{\prime}$, we see that $g \in \operatorname{Hom}_{D^{\prime}}\left(D^{\prime}, R^{\prime}\right)$. There exists a unique element $b \in P$ such that $b d^{\prime}=g\left(d^{\prime}\right)$ for all $d^{\prime} \in D^{\prime}$. For $r^{\prime} \in R^{\prime}, d^{\prime} \in D^{\prime}$, we have $b r^{\prime} d^{\prime}=b(d r)^{\prime}=g(d r)^{\prime}=(c d r)^{\prime}=r^{\prime}(c d)^{\prime}=r^{\prime} b d^{\prime}$, showing that $b r^{\prime}=r^{\prime} b$, i.e., $b \in B$. It can be verified that the mapping $c \rightarrow b$ is an isomorphism of $C$ onto $B$. Next suppose $\sum_{i=1}^{n} r_{i} c_{i}=0, r_{i} \in R, c_{i} \in C$. Let $D_{i}=\left\{x \in R \mid c_{i} x \in R\right\}$ and set $D=\bigcap D_{i}$. Then $D^{\prime}=\bigcap D_{i}^{\prime}$, and we see that, for $d^{\prime} \in D^{\prime},\left(\sum r_{i}^{\prime} b_{i}\right) d^{\prime}=$ $\sum r_{i}^{\prime}\left(b_{i} d^{\prime}\right)=\sum r_{i}^{\prime}\left(c_{i} d\right)^{\prime}=\left(\sum c_{i} d r_{i}\right)^{\prime}=\left(d \sum c_{i} r_{i}\right)^{\prime}=0$. Consequently $\sum r_{i}^{\prime} b_{i}=0$, which proves that the mapping $\chi: \sum r_{i} c_{i} \rightarrow \sum r_{i}^{\prime} b_{i}$ is well defined. It is then straightforward to verify that $\chi$ is an anti-isomorphism of $T=R C$ onto $U=R^{\prime} B$.

Lemma 22 may now be applied in order to write $\psi\left(e_{i}\right)=-\theta\left(\phi\left(e_{i}\right)\right)=-\theta\left(c_{i}-f_{i}\right)$ $=-\chi\left(c_{i}-f_{i}\right)=-\chi\left(c_{i}\right)+\chi\left(f_{i}\right)=b_{i}+g_{i}, i=1,2,\left\{g_{i}\right\}$ orthogonal idempotents of $U$. This shows that Case 1 arguments apply to $\chi$. By Theorems 9 and 10, therefore, $\psi=\sigma+\tau$, where $\sigma$ is either an isomorphism or the negative of an anti-isomorphism of $S$ into $U$ and $\tau$ is an additive mapping of $S$ into $B$ which maps commutators into zero. Hence $-\chi \phi=-\theta \phi=\sigma+\tau$, from which we obtain $\phi=-\chi^{-1} \sigma-\chi^{-1} \tau$ $=\sigma^{\prime}+\tau^{\prime}$, where $\sigma^{\prime}$ is either the negative of an anti-isomorphism or is an isomorphism of $S$ into $T$ and $\tau^{\prime}$ is an additive mapping of $S$ into $C$ which maps commutators into zero.

From Theorems 9 and 10 and their corollaries, and from the discussion of the preceding paragraph, it is clear that we have completed the proof of the main theorem. 
THEOREM 11. Let $S$ be a prime ring with 1 , of characteristic different from 2 and 3 , and containing two nonzero idempotents $e_{1}$ and $e_{2}$ whose sum is 1 . Let $\phi$ be a Lie isomorphism of $S$ onto a prime ring $R$ with 1 . Let $Q$ be the complete ring of right quotients of $R$, let $C$ be the center of $Q$, and let $T=R C$. Then $\phi$ is of the form $\sigma+\tau$, where $\sigma$ is either an isomorphism or the negative of an anti-isomorphism of $S$ into $T$ and $\tau$ is an additive mapping of $S$ into $C$ which maps commutators into zero.

It is only natural now to try to show that the results which we previously obtained for Lie isomorphisms in which the rings involved were either simple or primitive are actually corollaries of Theorem 11. Of course, since simple rings and primitive rings are necessarily prime rings, the obvious corollaries follow immediately. But sharper results are actually available, since in these special cases we now proceed to show that there is a close relationship between the ring $T=R C$ and the ring $R$. For simple rings, in fact, we prove that $T=R$.

LeMma 23. Let $R$ be a simple ring with 1 , with center $Z$, and let $Q$ be the complete ring of right quotients of $R$, with center $C$. Then $Z=C$ (and hence $T=R C=R$ ).

Proof. Let $c \in C$. The set $D=\{x \in R \mid c x \in R\}$, which we know is a dense right ideal of $R$, is clearly a two-sided ideal of $R$ and hence equal to $R$. In particular, $c=c \cdot 1 \in R$, and so $Z=C$.

Somewhat less obvious is the situation for primitive rings. Let $R$ be a primitive ring with 1 . We regard $R$ as an irreducible ring of endomorphisms of an additive abelian group $V$. The set $\Delta=\operatorname{Hom}_{R}(V, V)$ is a division ring, and we let $F$ denote the center of $\Delta . R F$ is a primitive ring with center $F$, and $R \subseteq R F$. On the other hand, if $Q$ is the complete ring of right quotients of $R$ and $C$ is the center of $Q$, we may still regard $R$ as a subring of $T=R C$.

THEOREM 12. $C$ is isomorphic to a subfield of $F$, and $R C$ is isomorphic to a subring of $R F$.

Proof. Let $c \in C$, and let $D=\{x \in R \mid c x \in R\}$. Choosing $v \neq 0 \in V$, we may write $V=v D$, since $D$ is an ideal of $R$. A mapping $f=f(c, v)$ of $V$ into $V$ is then defined by $v d \rightarrow v(c d)$ for all $d \in D$. Suppose $v d=0$. If $v(c d) \neq 0$, then $v(c d) D \neq 0$, and so there is an element $x \in D$ such that $v(c d) x \neq 0$. But $v(c d) x=v d(c x)=(v d)(c x)=0$, a contradiction. Therefore $v(c d)=0$, and $f$ is well defined. For $d \in D, r \in R$, we see that $(v d)(r f)=\{v(d r)\} f=v(c(d r))=v(c d) r=(v d) f r$, i.e., $f \in \Delta$. Now let $d \in D$, $a \in \Delta$, and write $v a=v x$ for some $x \in D$. Then $(v d)(a f)=(v a) d f=(v x) d f=v(x d) f$ $=v(c x d)=v x(c d)=(v a)(c d)=v(c d) a=(v d) f a$. Therefore $a f=f a$ and $f \in F$. We point out, too, that $f$ is independent of the choice of $v$. Indeed, suppose $v d=v_{1} d_{1}$, but $v(d c) \neq v_{1}\left(d_{1} c\right), v, v_{1} \in V, d, d_{1} \in D$. Then there is an $x \in D$ such that

$$
\left\{v(d c)-v_{1}\left(d_{1} c\right)\right\} x \neq 0 .
$$

But $v(d c) x=(v d)(c x)=\left(v_{1} d_{1}\right)(c x)=v_{1}\left(d_{1} c\right) x$, a contradiction. We are now able to map $C$ into $F$ by $\rho: c \rightarrow f=f(v, c)=f(c)$. For $c_{1}, c_{2} \in C$, let $D_{i}=\left\{x \in R \mid c_{i} x \in R\right\}$, 
$i=1,2$, set $D=D_{1} D_{2} \subseteq D_{1} \cap D_{2}$, and let $v \neq 0 \in V$. For $d \in D$, we have $(v d)\left(f\left(c_{1}+c_{2}\right)\right.$ $=\left(\left(c_{1}+c_{2}\right) d\right)=v\left(\left(c_{1} d\right)+\left(c_{2} d\right)\right)=v d f\left(c_{1}\right)+v d f\left(c_{2}\right)$. This shows that $\rho$ is additive. For $d \in D$, it is easy to see that $c_{2} d \in D_{1}$. Therefore, $(v d) f\left(c_{1} c_{2}\right)=v\left(c_{1} c_{2}\right) d=v c_{1}\left(c_{2} d\right)$ $=v\left(c_{2} d\right) f\left(c_{1}\right)=(v d) f\left(c_{2}\right) f\left(c_{1}\right)$. Hence $\rho$ is multiplicative. Suppose $f\left(c_{1}\right)=0$. Then $V\left(D_{1} c_{1}\right)=0$, and so $D_{1} c_{1}=0$, which implies that $c_{1}=0$. We have thus shown that $\rho$ is a ring isomorphism of $C$ into $F$. We define a mapping $\gamma$ of $R C$ into $R F$ according to

$$
\sum_{i=1}^{n} r_{i} c_{i} \rightarrow \sum_{i=1}^{n} r_{i} f_{i}
$$

where $f_{i}=\rho\left(c_{i}\right)$. Suppose $\sum r_{i} c_{i}=0$, but $\sum r_{i} f_{i} \neq 0$. Let $D_{i}=\left\{x \in R \mid c_{i} x \in R\right\}$, and set $D=\bigcap_{i=1}^{n} D_{i}$. For $v \in V$ and $d \in D$, we have

$$
(v d)\left(\sum r_{i} f_{i}\right)=\sum_{i} v\left(d r_{i}\right) f_{i}=\sum_{i} v\left(d r_{i}\right) c_{i}=v\left\{d\left(\sum r_{i} c_{i}\right)\right\}=0 .
$$

Hence $\sum r_{i} f_{i}=0$ and $\gamma$ is well defined. It is clear that $\gamma$ is a ring homomorphism, because $\rho$ is a homomorphism. Finally, suppose $\sum r_{i} f_{i}=0$. As above, let $D=\bigcap_{i=1}^{n} D_{i}$, let $v \in V$, and let $d \in D$. Then $0=v d\left(\sum r_{i} f_{i}\right)=v \sum\left(d r_{i}\right) c_{i}=v d\left(\sum r_{i} c_{i}\right)$. Therefore $\sum r_{i} c_{i}=0$ and $\gamma$ is one-one.

As a result of Theorem 11 and Theorem 12 we clearly have

THEOREM 13. Let $S$ be a prime ring with 1, of characteristic different from 2 and 3 , and containing two nonzero orthogonal idempotents whose sum is 1 . Let $\phi$ be a Lie isomorphism of $S$ onto a primitive ring $R$ with 1 . Let $R$ be regarded as an irreducible ring of endomorphisms of an additive abelian group $V$, let $\Delta$ be the division ring $\operatorname{Hom}_{R}(V, V)$, and let $F$ be the center of $\Delta$. Then $\phi$ is of the form $\sigma+\tau$, where $\sigma$ is either an isomorphism or the negative of an anti-isomorphism of $S$ into the primitive ring $R F$ and $\tau$ is an additive mapping of $S$ into $F$ which maps commutators into zero.

Except for the assumption that $1 \in R$, Theorem 13 is clearly a generalization of the main theorem of our first paper on Lie isomorphisms [2, p. 915, Theorem 4]. In that paper we assumed that $S$ was primitive and contained three nonzero orthogonal idempotents whose sum was 1 .

As a result of Theorem 11 and Lemma 23 (also as a corollary of Theorem 13), we have

THEOREM 14. Let $S$ be a prime ring with 1, of characteristic different from 2 and 3, and containing two nonzero orthogonal idempotents whose sum is 1 . Let $\phi$ be a Lie isomorphism of $S$ onto a simple ring $R$ with center $Z \neq 0$. Then $\phi$ is of the form $\sigma+\tau$, where $\sigma$ is either an isomorphism or the negative of an anti-isomorphism of $S$ onto $R$ (hence $S$ must be simple) and $\tau$ is an additive mapping of $S$ into $Z$ which maps commutators into zero.

Theorem 14 is the main theorem of our second paper on Lie isomorphisms [3]. 
Theorem 13 (and hence Theorem 11) has been illustrated with an example in [2, p. 916], which shows that the image of $\sigma$ need not be contained in $R$.

\section{BIBLIOGRAPHY}

1. L. Hua, A theorem on matrices over a sfield and its applications, J. Chinese Math. Soc. (N.S.) 1 (1951), 110-163.

2. W. S. Martindale III, Lie isomorphisms of primitive rings, Proc. Amer. Math. Soc. 14 (1963), 909-916.

3. - Lie isomorphisms of simple rings, J. London Math. Soc. 44 (1969), 213-221.

4. J. Lambek, Lectures on rings and modules, Blaisdell, Waltham, Mass., 1966.

5. S. A. Amitsur, Generalized polynomial identities and pivotal monomials, Trans. Amer. Math. Soc. 114 (1965), 210-226.

6. G. D. Findlay and J. Lambek, A generalized ring of quotients. I, II, Canad. Math. Bull. 1 (1958), 77-85, 155-167.

7. Y. Utumi, On quotient rings, Osaka J. Math. 8 (1956), 1-18.

8. I. N. Herstein, Topics in ring theory, Lecture notes, Sexto Coloquio Brasileiro de Matematica, July, 1967.

\section{UNIVERSITY OF MASSACHUSETTS,} AMHERST, MasSACHUSETtS 